Research Paper

\title{
Effects of dexmedetomidine in combination with fentanyl-based intravenous patient-controlled analgesia on pain attenuation after open gastrectomy in comparison with conventional thoracic epidural and fentanyl-based intravenous patient-controlled analgesia
}

Na Young Kim ${ }^{1}$, Tae Dong Kwon ${ }^{1}$, Sun Joon Bai ${ }^{1}$, Sung Hoon Noh², Jung Hwa Hong ${ }^{3}$, Haeyeon Lee ${ }^{1}$, and Ki-Young Lee ${ }^{1 凶}$

1. Department of Anesthesiology and Pain Medicine, Anesthesia and Pain Research Institute, Yonsei University College of Medicine, Seoul, Republic of Korea;

2. Department of Surgery, Yonsei University College of Medicine, Seoul, Republic of Korea;

3. Department of Policy Research Affairs National Health Insurance Service Ilsan Hospital, Goyang, Gyeonggi-do, Republic of Korea.

$\triangle$ Corresponding author: Ki-Young Lee, MD, PhD, Department of Anesthesiology and Pain Medicine, Anesthesia and Pain Research Institute, Yonsei University College of Medicine, 50-1 Yonsei-ro, Seodaemun-gu, Seoul 03722, Republic of Korea. Tel: (+82) 22228 4443; Fax: (+82) 222276517 E-mail: KYLEE504@yuhs.ac

(c) Ivyspring International Publisher. This is an open access article distributed under the terms of the Creative Commons Attribution (CC BY-NC) license (https://creativecommons.org/licenses/by-nc/4.0/). See http://ivyspring.com/terms for full terms and conditions.

Received: 2016.03.30; Accepted: 2017.06.18; Published: 2017.08.18

\begin{abstract}
Background: This study was investigated the effects of dexmedetomidine in combination with fentanyl-based intravenous patient-controlled analgesia (IV-PCA) on pain attenuation in patients undergoing open gastrectomy in comparison with conventional thoracic epidural patient-controlled analgesia (E-PCA) and IV-PCA.

Methods: One hundred seventy-one patients who planned open gastrectomy were randomly distributed into one of the 3 groups: conventional thoracic E-PCA (E-PCA group, $n=57)$, dexmedetomidine in combination with fentanyl-based IV-PCA (dIV-PCA group, $\mathrm{n}=57$ ), or fentanyl-based IV-PCA only (IV-PCA group, $n=57$ ). The primary outcome was the postoperative pain intensity (numerical rating scale) at 3 hours after surgery, and the secondary outcomes were the number of bolus deliveries and bolus attempts, and the number of patients who required additional rescue analgesics. Mean blood pressure, heart rate, and adverse effects were evaluated as well.

Results: One hundred fifty-three patients were finally completed the study. The postoperative pain intensity was significantly lower in the IIV-PCA and E-PCA groups than in the IV-PCA group, but comparable between the dIV-PCA group and the E-PCA group. Patients in the dIV-PCA and E-PCA groups needed significantly fewer additional analgesic rescues between 6 and 24 hours after surgery, and had a significantly lower number of bolus attempts and bolus deliveries during the first 24 hours after surgery than those in the IV-PCA group.

Conclusions: Dexmedetomidine in combination with fentanyl-based IV-PCA significantly improved postoperative analgesia in patients undergoing open gastrectomy without hemodynamic instability, which was comparable to thoracic E-PCA. Furthermore, this approach could be clinically more meaningful owing to its noninvasive nature.
\end{abstract}

Key words: dexmedetomidine, fentanyl, intravenous, epidural, patient-controlled analgesia, postoperative pain.

\section{Background}

Radical open gastrectomy is one of the major upper abdominal surgeries that have been reported to cause acute postoperative pain [1]. Moreover, the severity of pain is higher especially in this upper-abdominal surgery, which can lead to the impairment of the respiratory effort due to the restriction of the movement of the thoracic cage and 
abdomen, as well as the decreased respiratory capacity $[2,3]$. Such changes have a negative impact on the course of postoperative recovery [4].

Conventionally, pain after open gastrectomy has been controlled with thoracic epidural patient-controlled analgesia (E-PCA) or intravenous PCA (IV-PCA) [1, 4]. Thoracic E-PCA has an excellent effect in controlling postoperative pain, when properly positioned [1, 5-7]. However, as it is a relatively invasive technique, its application is limited by specific contraindications such as infection or bleeding tendency, and there is a possibility of malpositioning of the catheter in the spinal nerve roots leading to severe postoperative neurologic deficits due to ischemia of the sensory and motor nerves [5, 7-10]. Therefore, despite its potential benefits, the clinical use of E-PCA may have even declined because of these types of complications [1, 11].

In case of IV-PCA, higher doses of opioids are required to control postoperative pain effectively; however, this often leads to the discontinuation of IV-PCA because of persistent adverse effects such as nausea, vomiting, and pruritus $[1,12,13]$.

Dexmedetomidine is well recognized as an extremely preferential a2-receptor agonist that has sedative and analgesic effects without unfavorable respiratory suppression [14-16]. Previous studies have reported that dexmedetomidine administration during surgery could reduce the amounts of opioids and analgesics used after surgery [17-20]. Furthermore, current studies on the combination of dexmedetomidine with various opioid-based IV-PCA techniques have demonstrated that this combination treatment could help provide better analgesia and opioid-sparing effects without any remarkable unfavorable effects [21-24].

Hence, in this prospective, randomized clinical trial, we investigated the effects of dexmedetomidine in combination with fentanyl-based IV-PCA on pain attenuation in patients undergoing open gastrectomy in comparison with conventional thoracic E-PCA and IV-PCA.

\section{Materials and Methods}

\section{Study population}

This investigation was approved from the Institutional Review Board and Hospital Research Ethics Committee of Severance Hospital (Yonsei University Health System in Seoul, Korea; IRB protocol No. 4-2014-0883), and consequently registered at http:/ / clinicaltrials.gov (registration No. NCT02325882). After acquiring written informed consent from all patients, 171 patients with stomach cancer, of age 20 to 65 years and American Society of Anesthesiologists physical status I/II, who were planned to undergo elective conventional open gastrectomy, were enrolled between July, 2015 and March, 2016. The exclusion criteria were as follows: refusal of PCA application; histories of abdominal surgery; prior cardiac disease including unstable angina, congestive heart failure, uncontrolled hypertension; concomitant coagulopathy; presence of vertebral deformity or disease; concomitant pulmonary, renal, or hepatic disease; any contraindication to epidural catheterization; any allergy or hypersensitivity to fentanyl, $\mathrm{a}_{2}$-adrenergic agonists, or local anesthetics; use of any type of chronic pain killer or current opioid; cognitive, neurological, or psychiatric impairment; and incapability to report the pain intensity on the pain scale. All enrolled patients were educated on how to express the intensity of pain by using the numerical rating scale (NRS; 0 , no pain, and 10, worst pain possible) [25], and on how to use the PCA machine in the preanesthetic room.

\section{Randomization and Perioperative Protocol}

The assignments of the patients were performed randomly into one of 3 groups (1:1:1) according to preset random numbers by using a computer-generated table (http://www.random.org) with no dividing blocks and stratification: conventional thoracic E-PCA (E-PCA group, $\mathrm{n}=57$ ), dexmedetomidine in combination with fentanyl-based IV-PCA (dIV-PCA group, $\mathrm{n}=57$ ), or fentanyl-based IV-PCA only (IV-PCA group, $\mathrm{n}=57$ ).

In the E-PCA group, the procedure for epidural catheter insertion was completed before the induction of general anesthesia. After standard monitoring, a single investigator performed the epidural catheterization at the level of T7-8 or T8-9 by using a 17-gauge Tuohy needle, and a catheter was advanced $5 \mathrm{~cm}$ into the epidural space. Intravascular or subarachnoid placement of the epidural catheter was excluded by checking the absence of aspirated blood or cerebrospinal fluid. Furthermore, intrathecal delivery of the local anesthetic was ruled out by confirming that no rapid onset of neuroaxial block was developed after the administration of $3 \mathrm{~mL}$ of $1 \%$ lidocaine. Upon the initiation of peritoneal closure, the PCA machine (Accumate 1100 ${ }^{\circledR}$; Woo Young Medical Co., Ltd., Seoul, Korea) was started after 5 $\mathrm{mL}$ of $0.15 \%$ ropivacaine was administered via the epidural catheter. The PCA regimen was a mixture of $0.15 \%$ ropivacaine and $3 \mu \mathrm{g} / \mathrm{mL}$ of fentanyl in $0.9 \%$ normal saline solution with a total volume of $250 \mathrm{~mL}$. All PCA machines for the 3 groups were programmed to deliver at the rate of $5 \mathrm{~mL} / \mathrm{h}$ with a $0.5 \mathrm{~mL}$ per 
demand allowed every 15-minute lockout time.

In the IV-PCA group, after $1 \mu \mathrm{g} / \mathrm{kg}$ of fentanyl was administered intravenously at the start of peritoneal closure, PCA machine was applied intravenously, which consisted $15 \mu \mathrm{g} / \mathrm{kg}$ of fentanyl and $0.3 \mathrm{mg}$ of ramosetron (Nasea, Astellas, Tokuo, Japan), mixed with $0.9 \%$ normal saline solution to a total volume of $250 \mathrm{~mL}$. Thus, in the IV-PCA group, fentanyl was infused basally at a rate of $0.3 \mu \mathrm{g} / \mathrm{kg} / \mathrm{h}$ with a bolus dose of $0.03 \mu \mathrm{g} / \mathrm{kg}$ and a lockout time of 15-min.

In the dIV-PCA group, dexmedetomidine (100 $\mu \mathrm{g} / \mathrm{mL}$ at $2 \mathrm{~mL} / \mathrm{vial}$; Hospira Worldwide, Seoul, Korea) was infused continuously at a rate of 0.1 $\mu \mathrm{g} / \mathrm{kg} / \mathrm{h}$ from anesthetic induction until the start of peritoneal closure. Subsequently, the PCA, containing dexmedetomidine in addition to the fentanyl and ramosetron like in the IV-PCA group, was applied intravenously. Thus, in the dIV-PCA group, the background infusion rate of dexmedetomidine was $0.07 \mu \mathrm{g} / \mathrm{kg} / \mathrm{h}$ with a bolus dose of $0.007 \mu \mathrm{g} / \mathrm{kg}$, and that of fentanyl was $0.3 \mu \mathrm{g} / \mathrm{kg} / \mathrm{h}$ with a bolus dose of $0.03 \mu \mathrm{g} / \mathrm{kg}$ allowed every 15-min lockout time. In all three groups, the agents for PCA and the study drug were prepared by an investigator who was not involved in the assessment of postoperative pain intensity.

\section{Anesthesia}

Anesthesia was accomplished along with the same standard protocol in all three groups. After the patient arrived in the operating room, premedication was done with $0.1 \mathrm{mg}$ of glycopyrrolate administered intravenously. All patients were applied with noninvasive arterial blood pressure monitoring device for mean blood pressure (MBP) measurement, electrocardiogram (ECG) for heart rate (HR) monitoring, oxygen saturation $\left(\mathrm{SpO}_{2}\right)$ measurement device, and bispectral index (BIS) monitor (Aspect A-2000 ${ }^{\circledR}$; Aspect Medical System Inc., Newton, MA, USA). Anesthesia was induced with $1.5 \mathrm{mg} / \mathrm{kg}$ of propofol, $0.5 \mu \mathrm{g} / \mathrm{kg}$ of remifentanil, and $1.2 \mathrm{mg} / \mathrm{kg}$ of rocuronium. Thereafter, mechanical ventilation was kept to maintain the end-tidal carbon dioxide at 30-40 $\mathrm{mm} \mathrm{Hg}$ in $50 \% \mathrm{O}_{2} /$ air throughout the surgery. Anesthesia was maintained with 0.6-1.2 age-adjusted minimal alveolar concentration end-tidal sevoflurane and $0.02-0.2 \mu \mathrm{g} / \mathrm{kg} / \mathrm{min}$ of remifentanil, which were adjusted according to stable hemodynamic variables, including MBP or HR maintained within $20 \%$ of the baseline and BIS scores between 40 and 60 . Hypotension [MBP $<60 \mathrm{~mm} \mathrm{Hg}$ or systolic blood pressure (SBP) $<90 \mathrm{~mm} \mathrm{Hg}$ ] was managed with fluid loading at $100 \mathrm{~mL}$ increments or intravenous ephedrine at $4 \mathrm{mg}$ increments, and $0.25 \mathrm{mg}$ intravenous atropine was used to manage bradycardia (HR $<40$ beats/min). For the prevention of postoperative nausea and vomiting (PONV), 0.3 $\mathrm{mg}$ of ramosetron was administered at the start of peritoneal closure, and naloxone and oxygen were prepared for the event of respiratory depression. In case of the development of persistent complications such as severe PONV, hypotension, bradycardia, and respiratory depression despite of appropriate treatment, applications with the PCA machine were discontinued.

\section{Data Collection}

When the patients were transferred to the postanesthesia care unit (PACU) after surgery, they were reinstructed about the use of the PCA machine. Thereafter, recovery nurses who were not involved in this study assessed the resting NRS scores at $0.5 \mathrm{~h}$ and encouraged the patients to push the bolus button whenever they feel pain at a resting NRS score of $>3$. For patients who showed poor response to the PCA, thus felt sustained pain at a resting NRS score of $>4$, additional rescue analgesics with pethidine at $12.5 \mathrm{mg}$ increments were given. After PACU discharge, postoperative pain assessment was performed at 1, 2, $3,6,12,18,24$, and $36 \mathrm{~h}$ after surgery by the attending nurses of the Postoperative PCA Management Services of our institution, who were not aware of the purpose of this study. Similarly, for patients who experienced sustained pain at a resting NRS score of $>4$ in the admission room, additional rescue analgesics of pethidine at $12.5 \mathrm{mg}$ increments were also administered. After finishing the infusion of PCA, the machine was taken off and sent to the anesthesiology department for the evaluation of all records in relation to the deliveries and attempts with the bolus button. In addition to the records of the PCA machine, the number of patients who required additional rescue analgesics was also noted. MBP and HR data were collected at baseline; at PACU arrival; and at $0.5,1,2,3,6,12,18,24$, and $36 \mathrm{~h}$ after surgery. The level of sedation (assessed on a 5-point scale-0, fully awake; 1 , drowsy/closed eyes; 2 , asleep/easily aroused with light tactile stimulation or a simple verbal command; 3, asleep/arousable only with strong physical stimulation; and 4, unarousable) was assessed as well.

\section{Statistical Analysis}

On the basis of a preliminary study, the mean \pm standard deviation (SD) of the resting NRS score at $3 \mathrm{~h}$ after surgery in the IV-PCA group was 5.35, and the corresponding value for the E-PCA group was 4.38. In order to detect an expected difference of 1 with a SD of 1.8 for the resting NRS score in the dIV-PCA, the 
obtained sample size in each group was 51 patients with $\alpha=0.05$ and $\beta=0.8$. Assuming a possible dropout rate of $10 \%$, 57 patients were determined to be required in each group.

Statistical analyses were performed by using SAS software version 9.2 (SAS Inc., Cary, NC, USA) and IBM SPSS Statistics 20 (SPSS Inc., Chicago, IL, USA). All values were shown as mean $\pm S D$, number of patients (proportion), or median (range). One-way analysis of variance was performed to analyze all parametric variables among the three groups, and nonparametric data were analyzed by using the Kruskal-Wallis test. For categorical data, the Chi-square test or Fisher's exact test was used in the analysis when applicable. A linear mixed model was used in the analysis for repeated-measure variables such as NRS, MBP, and HR. Post-hoc analyses with Bonferroni correction were applied when the interaction of group, time, and group by time showed statistical significance. A $P$ value of $<0.05$ was taken to indicate statistical significance.

\section{Results}

Of 190 patients evaluated for eligibility, 171 patients were initially registered and assigned into the 3 groups. Ten patients in the E-PCA group were eliminated because PCA was discontinued owing to persistent hypotension. In the dIV-PCA group, 3 patients were excluded from the analysis for the following reasons: one patient did not receive the allocated intervention because of another surgery, one patient discontinued PCA because of persistent dizziness, and one patient had deleted PCA data due to a mechanical problem of the PCA machine. Five patients in the IV-PCA group were removed from the analysis for the following reasons: one patient did not receive the allocated intervention because of another surgery, three patients discontinued PCA because of persistent nausea, and one patient had deleted PCA data due to a mechanical problem of the PCA machine. The remaining 153 patients successfully completed the study without any complications (Figure 1).

The demographic and intraoperative variables were shown (Table 1). Apart from the total administered dose of remifentanil and ephedrine, there were no significant differences among the 3 groups. The total administered dose of remifentanil was higher in the IV-PCA group than in the E-PCA and dIV-PCA groups (Bonferroni corrected $P=0.017$ and $P<0.001$, respectively). In addition, the patients in the E-PCA group required more ephedrine than those in the IV-PCA group (8.4 \pm 9.1 vs. $4.0 \pm 4.8 \mu \mathrm{g}$; Bonferroni corrected $P=0.013)$.

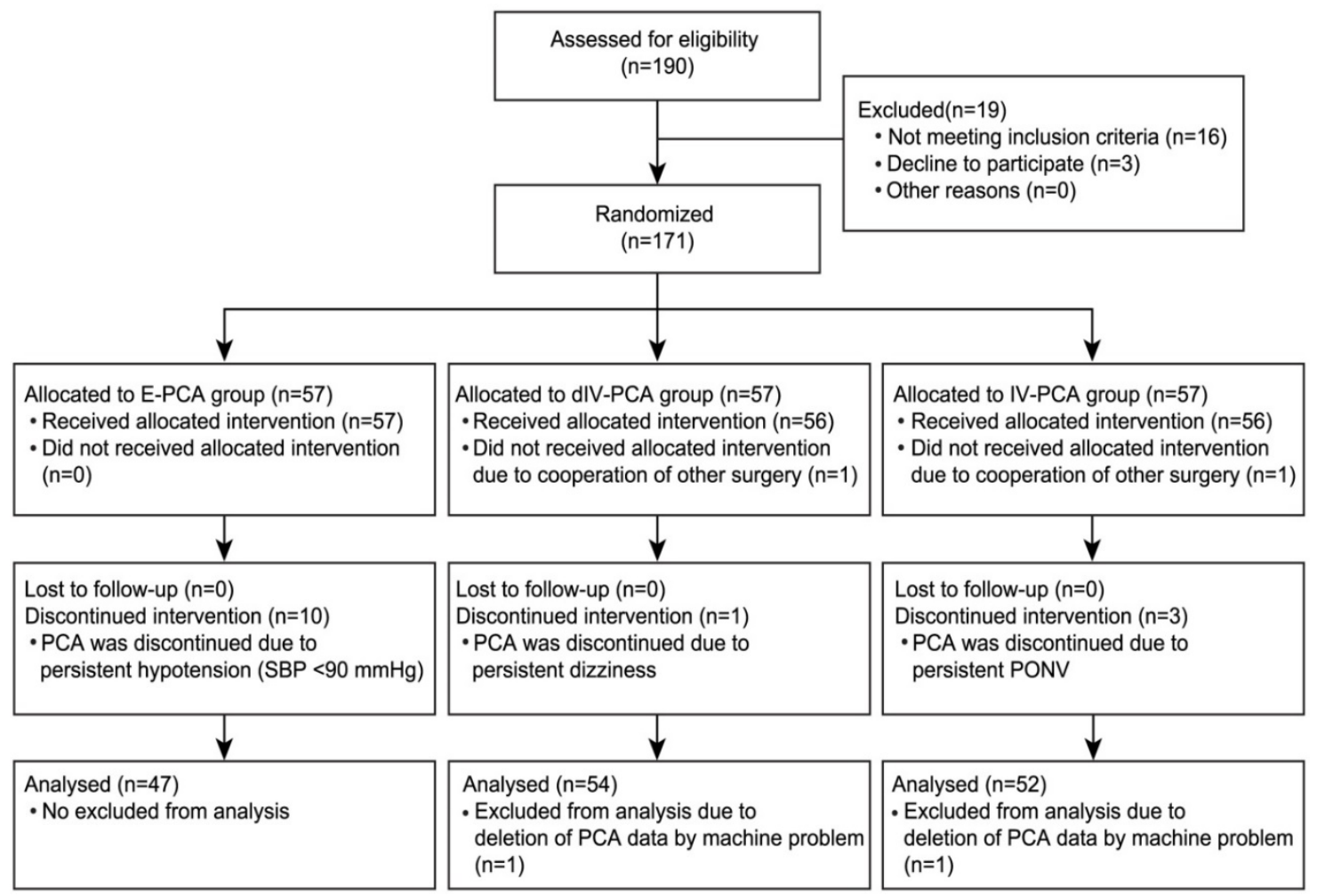

Figure 1. Consort flow diagram. E-PCA, epidural patient-controlled analgesia; dIV-PCA, dexmedetomidine in combination with fentanyl-based intravenous patient-controlled analgesia; IV-PCA, intravenous patient-controlled analgesia; SBP, systolic blood pressure; PONV, postoperative nausea and vomiting. 
The pain scores at rest were shown in Figure 2. Postoperative pain intensity was significantly lower in the dIV-PCA and E-PCA groups than in the IV-PCA group, however, it was comparable between the dIV-PCA group and the E-PCA group. After post-hoc analysis with Bonferroni corrections, the NRS scores for resting pain in the dIV-PCA group were lower than those in the IV-PCA group at all time points during the $36 \mathrm{~h}$ after surgery $(P<0.01$, Bonferroni corrected), and the E-PCA group showed lower NSR scores than those in the IV-PCA group at $0.5,2,3,6,12,18,24$, and 36 h after surgery $(P<0.01$, Bonferroni corrected). Moreover, patients of the dIV-PCA group required significantly fewer additional analgesic rescues than did patients of the IV-PCA group between 2 and $24 \mathrm{~h}$ after surgery, and patients in the E-PCA group needed significantly fewer additional analgesic rescues than those in the IV-PCA group between 6 and $24 \mathrm{~h}$ after surgery (Table 2). Figure 3 showed the number of bolus attempts and the number of successful bolus deliveries during the first $36 \mathrm{~h}$ after surgery. Patients in the dIV-PCA and E-PCA groups had a significantly lower number of bolus attempts and bolus deliveries than those in the IV-PCA group during the first $24 \mathrm{~h}$ after surgery (both $P<0.05$, Bonferroni corrected).

Significant differences in MBP and HR were observed among groups in the linear mixed model analysis $(P=0.007$ and $P<0.001$, respectively) (Figure 4). MBP in the E-PCA group was lower than that in the IV-PCA group at 3, 12, and $18 \mathrm{~h}$ after surgery, although more ephedrine was administered in the E-PCA group than in the IV-PCA group $(P=0.023$, 0.010 , and 0.033, respectively; Bonferroni corrected). Furthermore, patients in the dIV-PCA group showed lower MBP than those in the IV-PCA group at 1, 3, 6,
$12,18,24$, and $36 \mathrm{~h}$ after surgery $(P<0.05$, Bonferroni corrected). HR was lower in the dIV-PCA group than in the E-PCA group at 2,3 , and $6 \mathrm{~h}$ after surgery $(P=$ $0.02,0.01$, and 0.02, respectively; Bonferroni corrected). However, no patient in either group required atropine administration. The other postoperative adverse effects were not significantly different among the 3 groups $(P>0.05$; Table 3$)$. In addition, there were no patients who exhibited respiratory depression.

Table 2. Number of Patients Who Needed Additional Rescue Analgesics (Pethidine) During $36 \mathrm{~h}$ After Surgery

\begin{tabular}{lllll}
\hline Interval & $\begin{array}{l}\text { E-PCA group } \\
(\mathrm{n}=47)\end{array}$ & $\begin{array}{l}\text { dIV-PCA group } \\
(\mathrm{n}=54)\end{array}$ & $\begin{array}{l}\text { IV-PCA group } \\
(\mathrm{n}=52)\end{array}$ & $\begin{array}{l}\mathrm{P} \\
\text { value }\end{array}$ \\
\hline $0-2 \mathrm{~h}$ & $16(34 \%)$ & $22(41 \%)$ & $28(54 \%)$ & 0.054 \\
$2-6 \mathrm{~h}$ & $12(26 \%)$ & $10(19 \%)^{*}$ & $23(44 \%)$ & 0.012 \\
$6-12 \mathrm{~h}$ & $9(19 \%)^{\dagger}$ & $8(15 \%)^{*}$ & $24(46 \%)$ & $<0.001$ \\
$12-24 \mathrm{~h}$ & $12(26 \%)^{\dagger}$ & $12(22 \%)^{*}$ & $33(63 \%)$ & $<0.001$ \\
$24-36 \mathrm{~h}$ & $6(13 \%)$ & $4(7 \%)$ & $10(19 \%)$ & 0.199 \\
\hline
\end{tabular}

Data are presented as number of patients (proportion).

$\dagger \mathrm{P}<0.01$, vs. IV-PCA group (Bonferroni corrected), ${ }^{*} \mathrm{P}<0.01 \mathrm{vs}$. IV-PCA group (Bonferroni corrected)

Table 3. Postoperative Adverse Effects

\begin{tabular}{lllll}
\hline & $\begin{array}{l}\text { E-PCA } \\
(\mathrm{n}=47)\end{array}$ & $\begin{array}{l}\text { dIV-PCA } \\
(\mathrm{n}=54)\end{array}$ & $\begin{array}{l}\text { IV- PCA } \\
(\mathrm{n}=52)\end{array}$ & P value \\
\hline Sedation scores & $0(0-0)$ & $0(0-1)$ & $0(0-1)$ & 0.41 \\
Nausea & 5 & 6 & 7 & 0.904 \\
Dizziness & 1 & 4 & 3 & 0.594 \\
Headache & 1 & 3 & 2 & 0.056 \\
Hypotensive episode & 4 & 3 & 1 & 0.354 \\
Urinary retention & 7 & 5 & 5 & 0.653
\end{tabular}

Data are presented as median (interquartile range) or number of patients. Level of sedation; 0 = fully awake, $1=$ drowsy/closed eyes, $2=$ asleep/easily aroused with light tactile stimulation or a simple verbal command, $3=$ asleep/arousable only by strong physical stimulation, and $4=$ unarousable.

Table 1. Patient Characteristics and Intraoperative Variables

\begin{tabular}{|c|c|c|c|c|}
\hline & $\begin{array}{l}\text { E-PCA group } \\
(\mathrm{n}=47)\end{array}$ & $\begin{array}{l}\text { dIV-PCA group } \\
(n=54)\end{array}$ & $\begin{array}{l}\text { IV- PCA group } \\
(\mathrm{n}=52)\end{array}$ & $P$ value \\
\hline Age, years & $58 \pm 12$ & $59 \pm 7$ & $62 \pm 13$ & 0.148 \\
\hline Height, $\mathrm{cm}$ & $167 \pm 9$ & $164 \pm 8$ & $163 \pm 8$ & 0.110 \\
\hline Weight, kg & $64 \pm 11$ & $62 \pm 9$ & $61 \pm 12$ & 0.558 \\
\hline ASA physical status, I/II & $18 / 29$ & $23 / 31$ & $20 / 32$ & 0.895 \\
\hline Hypertension & $17(36 \%)$ & $19(35 \%)$ & $20(39 \%)$ & 0.955 \\
\hline Diabetes mellitus & $3(6 \%)$ & $5(9 \%)$ & $6(12 \%)$ & 0.686 \\
\hline Female gender & $16(34 \%)$ & $20(37 \%)$ & $20(39 \%)$ & 0.892 \\
\hline Subtotal/Total & $32 / 15$ & $36 / 18$ & $35 / 17$ & 1.000 \\
\hline Duration of surgery, min & $179 \pm 41$ & $170 \pm 32$ & $178 \pm 43$ & 0.500 \\
\hline Fluid intake, $\mathrm{mL}$ & $1743 \pm 468$ & $1717 \pm 489$ & $1795 \pm 744$ & 0.783 \\
\hline Blood loss, mL & $223 \pm 145$ & $213 \pm 182$ & $231 \pm 166$ & 0.859 \\
\hline Urine output, $\mathrm{mL}$ & $241 \pm 118$ & $238 \pm 171$ & $276 \pm 198$ & 0.450 \\
\hline Administered dose of remifentanil, $\mu \mathrm{g}$ & $814 \pm 280^{\dagger}$ & $660 \pm 260^{*}$ & $1000 \pm 400$ & $<0.001$ \\
\hline Administered dose of ephedrine, $\mathrm{mg}$ & $8.4 \pm 9.1 \ddagger$ & $6.3 \pm 7.5$ & $4.0 \pm 4.8$ & 0.016 \\
\hline
\end{tabular}




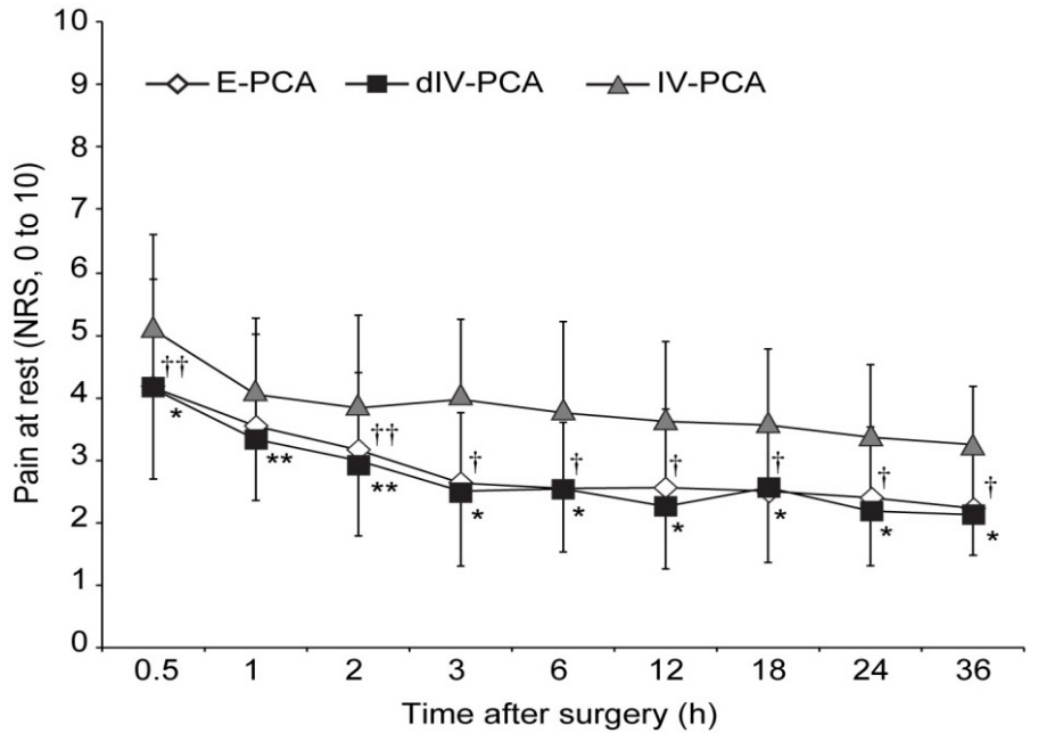

Figure 2. Pain score at rest during the first $36 \mathbf{~ h}$ after surgery. Data are expressed as mean \pm standard deviation. $t P<0.001$, $t+P<0.01$ vs. the IV-PCA group (Bonferroni corrected); $* P<0.001$, $* * P<0.01$ vs. the IV-PCA group (Bonferroni corrected). E-PCA, epidural patient-controlled analgesia; dIV-PCA, dexmedetomidine in combination with fentanyl-based intravenous patient-controlled analgesia; IV-PCA, intravenous patient-controlled analgesia; NRS, numerical rating scale.
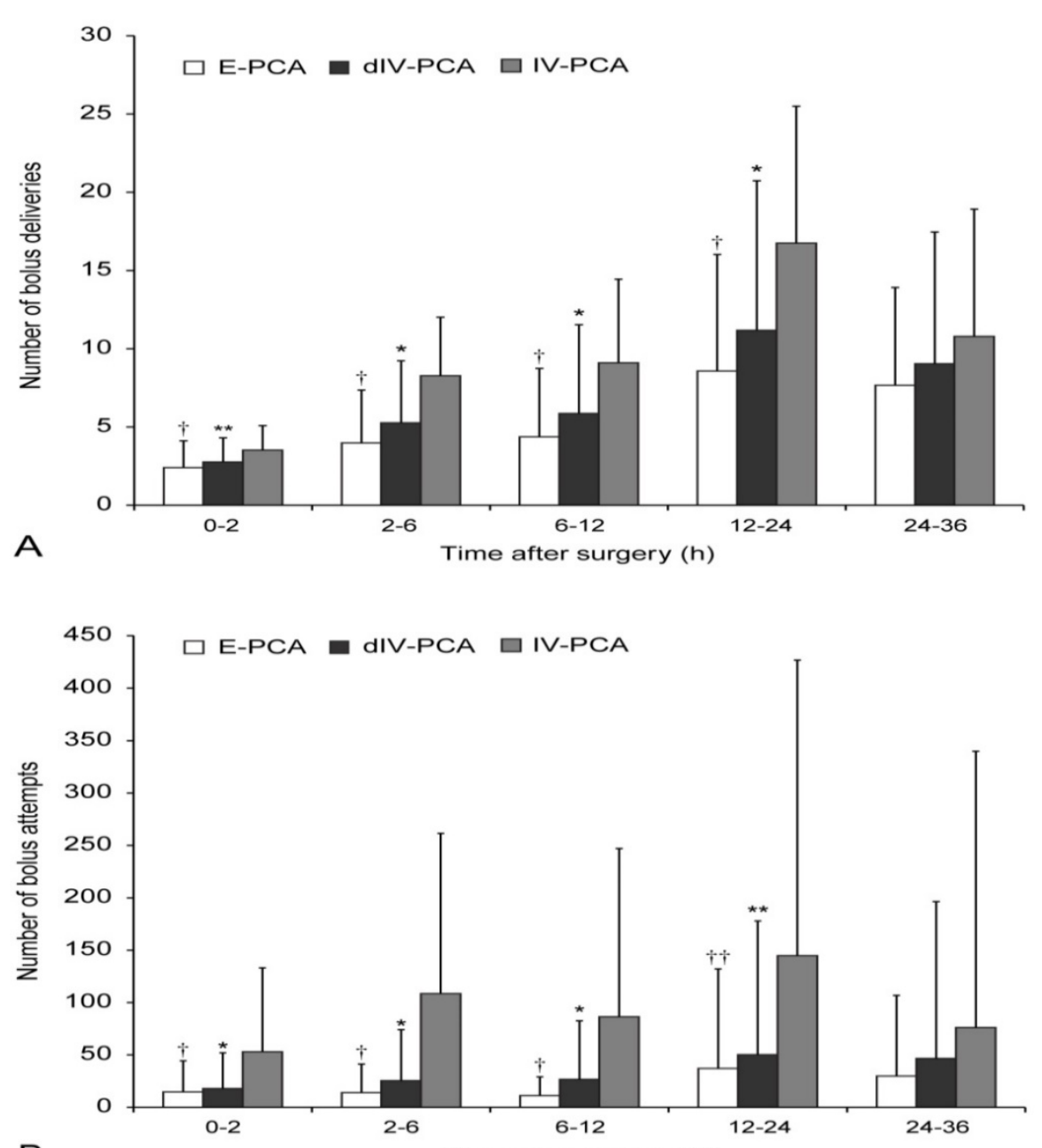

B

Time after surgery $(\mathrm{h})$

Figure 3. Number of bolus deliveries (A) and the number of bolus attempts (B) during the first $36 \mathrm{~h}$ after surgery. Data are expressed as mean \pm standard deviation. $t P<0.01,+t P<0.05$ vs. the IV-PCA group (Bonferroni corrected); $* P<0.01, * * P<0.05$ vs. the IV-PCA group (Bonferroni corrected). E-PCA, epidural patient-controlled analgesia; dIV-PCA, dexmedetomidine in combination with fentanyl-based intravenous patient-controlled analgesia; IV-PCA, intravenous patient-controlled analgesia 

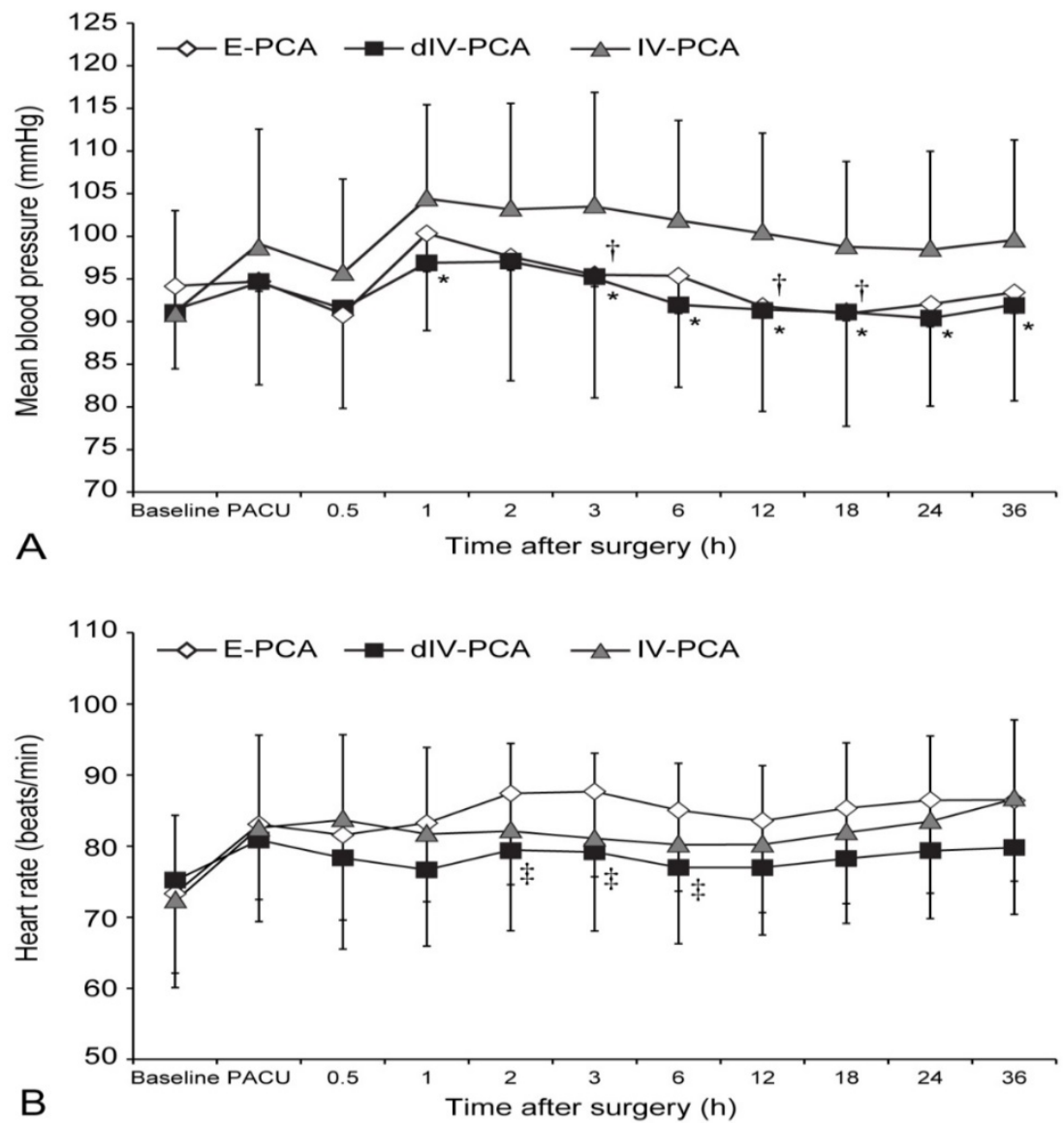

Figure 4. Mean blood pressure (A) and heart rate (B) from prior induction until $36 \mathbf{h}$ after surgery. Data are expressed as mean \pm standard deviation. $* P<0.05,+P<0.05$ vs. the IV-PCA group (Bonferroni corrected); $\neq P<0.05$ vs. the E-PCA group (Bonferroni corrected). E-PCA, epidural patient-controlled analgesia; dIV-PCA, dexmedetomidine in combination with fentanyl-based intravenous patient-controlled analgesia; IV-PCA, intravenous patient-controlled analgesia; Baseline, before induction of anesthesia; PACU, on arrival of post-anesthetic care unit.

\section{Discussion}

This prospective randomized study demonstrated that for patients undergoing open gastrectomy, dexmedetomidine in combination with fentanyl-based IV-PCA significantly improved postoperative analgesia than fentanyl-based IV-PCA, which was comparable to thoracic E-PCA. Furthermore, such improved effects could be achieved without hemodynamic instability by using this dexmedetomidine-fentanyl combination as a noninvasive treatment.

It is generally recognized that intense pain occurring during the postoperative period may have a major impact on the postoperative clinical outcomes. Insufficient analgesia might cause psychological distress as well as physical impairment, several postoperative complications, and even progression to chronic pain $[2,26]$. Especially, pain after the major abdominal surgery such as open gastrectomy could lead to restriction of thoracic and abdominal respiration as well as attenuation of vital capacity and tidal volume breathing, which probably have adverse effects on the respiratory drive [27, 28]. In addition, it may result in significant cardiovascular changes, cognitive impairment, delayed recovery of bowel motility, and neuroendocrine instability, which will most likely have a deleterious effect on the postoperative recovery process [4]. Thus, postoperative pain management concomitant with maintenance of hemodynamic stability is very crucial. In the last few decades, thoracic E-PCA and IV-PCA have been generally used for postoperative analgesia in patients after open gastrectomy [1, 4]. Several studies have reported that thoracic E-PCA is considered more effective than IV-PCA in relieving postoperative pain $[4,29,30]$. Furthermore, current research indicates that thoracic E-PCA is considered the "golden" standard in the management of pain after the major upper abdominal surgery, owing to its excellent analgesic effects [1,5-7]. However, it is a relatively invasive technique and its application is limited by specific contraindications such as infection or bleeding tendency [7]. In addition to these limitations, there is a possibility of several complications such as hematoma, or severe postoperative neurologic deficits resulting from 
malpositioning of the catheter in the spinal nerve roots $[8,9]$. Therefore, despite its potential benefits, the clinical use of E-PCA may have even declined because of these types of complications $[1,11]$.

IV-PCA requires a higher dose of opioids in order to acquire satisfactory analgesic effects. This, in turn, produces adverse effects such as nausea, vomiting and pruritus, which causes patients to discontinue the use of intravenous PCA $[1,12,13]$. Indeed, in the present study, 3 patients in the IV-PCA group chose to discontinue the use of PCA because of persistent PONV. For postoperative recovery, it is very crucial to amplify pain relief without increasing the adverse effects of analgesics. The multimodal analgesic approach, which involves using analgesics with different action mechanisms, might be a good strategy in the current setting [31,32]. Of the various available multimodal protocols, the combination of an opioid with one or more adjunctive drugs, such as nonsteroidal anti-inflammatory agents, pure opioid antagonists, and ketamine, has been considered the expedient option for IV-PCA in current postoperative pain management [33-35].

Dexmedetomidine, an extremely selective $\mathrm{a}_{2}$-adrenergic agonist that has hypnotic, sedative, and analgesic actions and generates sympatholytic responses, does not cause unfavorable respiratory suppression [14-16]. Currently, it has been suggested that combination treatment with dexmedetomidine and opioid-based IV-PCA could provide better analgesic and opioid-sparing effects without any remarkable detrimental influences [21-24]. However, to the best of our knowledge, no prior studies have investigated the impact of dexmedetomidine in combination with fentanyl-based IV-PCA on the attenuation of postoperative pain intensity in comparison with thoracic E-PCA and IV-PCA.

In the present study, we found significantly reduced resting NRS scores in the dIV-PCA group compared with those in the IV-PCA group during the first $36 \mathrm{~h}$ after surgery, although the number of bolus deliveries and attempts was significantly lower in the dIV-PCA group than in the IV-PCA group for the first $24 \mathrm{~h}$ after surgery; this finding was in accordance with those of previous reports [21-24]. Moreover, patients in the dIV-PCA group required significantly fewer additional rescue analgesics during 2-6, 6-12, and 12-24 h after surgery than those in the IV-PCA group $\left({ }^{*} P=0.004, P<0.001, P<0.001\right.$, respectively; Bonferroni corrected). In the dIV-PCA group in comparison with the E-PCA group, comparable analgesic effects were achieved. A tendency was shown that the number of bolus deliveries and attempts were lower in patients of the E-PCA group than those in patients of the dIV-PCA group; however, no statistical difference was observed after post-hoc analysis with Bonferroni correction.

Epidural-induced hypotension is also very common, which is partly due to cardio-depressant activity and arteriovenous vasodilation [7, 36, 37]. In the present study, persistent hypotension (SBP $<90$ $\mathrm{mm} \mathrm{Hg}$ ) developed in 10 patients of the E-PCA group. Consequently, these patients were excluded because of the discontinuation of use of the PCA machine (Figure 1). Except for the 10 patients who were dropped from the E-PCA group, none of the patients in all groups developed severe hemodynamic instability (SBP $<90 \mathrm{~mm} \mathrm{Hg}$, MBP $<60 \mathrm{~mm} \mathrm{Hg}$ ). Previous trials have been conducted with various dosages for an infusion rate of dexmedetomidine in PCA mixture from 0.02 to $0.6 \mu \mathrm{g} / \mathrm{kg} / \mathrm{h}$ within the range of the recommended dose by the manufacturer $(0.2-0.7 \mu \mathrm{g} / \mathrm{kg} / \mathrm{h})[21-24,38]$. In the present study, we selected $0.07 \mu \mathrm{g} / \mathrm{kg} / \mathrm{h}$ as the infusion dose and $0.007 \mu \mathrm{g} / \mathrm{kg} / \mathrm{h}$ as the bolus dose with a maximum limit of $0.1 \mu \mathrm{g} / \mathrm{kg} / \mathrm{h}$ in order to acquire the postoperative analgesic effect concomitant with maintaining hemodynamic stability. $\mathrm{MBP}$ in the dIV-PCA group were significantly lower than those in the IV-PCA group at 1 and 3-36 $\mathrm{h}$ after surgery; however, at all time points, the MBP in the dIV-PCA group were $>65 \mathrm{~mm} \mathrm{Hg}$. The patient who showed the lowest MBP was in the E-PCA group, which was 61 $\mathrm{mmHg}$. Furthermore, 4 patients in the E-PCA group and 3 patients in the dIV-PCA group developed intermittent mild hypotension (SBP $<100 \mathrm{~mm} \mathrm{Hg}$ ), with no statistical difference. Moreover, no bradycardia (HR $<40$ beats/min) that had to be treated with atropine occurred in all of the 3 groups. Thus, these study findings may have clinical implication, considering that low dose of dexmedetomidine-fentanyl combination significantly improved postoperative analgesia while maintaining stable hemodynamics; especially for those patients who have limitations in applying the E-PCA.

In addition, no significant difference was detected in postoperative adverse effects among the 3 groups $(P>0.05)$. The incidence of PONV in our trials was not consistent with the findings of previous reports [21, 38]. This discrepancy might be derived from the low doses of dexmedetomidine (infusion rate, $0.07 \mu \mathrm{g} / \mathrm{kg} / \mathrm{h}$; bolus rate, $0.007 \mu \mathrm{g} / \mathrm{kg} / \mathrm{h}$; maximum limit, $0.1 \mu \mathrm{g} / \mathrm{kg} / \mathrm{h}$ ) used in this study. Moreover, it might also be attributed to the removal of 3 patients from the IV-PCA group because of persistent PONV.

This study has several limitations. First, the patients received three different PCA regimens via different routes in accordance with the group allocation. However, we did not control this 
confounding factor because the objective of our study was to investigate the effect of dexmedetomidine in combination with IV-PCA on pain intensity compared with the standard methods and regimens of PCA. Second, it still needs to be clarified whether the effects of dexmedetomidine in combination with IV-PCA on pain attenuation, compared with those of E-PCA, are dose dependent. In addition, more long-term follow-up data are required to evaluate the effects of dexmedetomidine-opioid combination on postoperative outcomes, including chronic pain. Thus, further investigations are imperative. Third, we included patients with a wide age range (20 to 65 years), who underwent two types of surgeries (subtotal or total gastrectomy). Although the extent of postoperative pain intensity varies depending on the age, sex, and type of surgeries, the similar demographic variables among the 3 groups in the present study may have helped in preventing these variables from affecting the results of this study. Finally, it is uncertain whether the effects of dexmedetomidine on the attenuation of pain intensity were due to analgesic effect of itself or an indirect effect that decrease the remifentanil-induced hyperalgesia by reducing intraoperative remifentanil amounts. Therefore, more studies performed in regard to various setting would be needed.

\section{Conclusions}

Dexmedetomidine in combination with fentanyl-based IV-PCA significantly improved postoperative analgesia in patients undergoing open gastrectomy than fentanyl-based IV-PCA alone, comparable to thoracic E-PCA. Such improved effects could be achieved without hemodynamic instability; furthermore, this approach could be clinically more meaningful owing to its noninvasive nature.

\section{Abbreviations}

dIV-PCA, dexmedetomidine in combination with fentanyl-based intravenous patient-controlled analgesia

E-PCA, epidural patient-controlled analgesia

$\mathrm{HR}$, heart rate

IV-PCA, intravenous patient-controlled analgesia

$\mathrm{MBP}$, mean blood pressure

NRS, numerical rating scale

PACU, post-anesthesia care unit

PCA, patient-controlled analgesia

PONV, postoperative nausea and vomiting

$\mathrm{SD}$, standard deviation

\section{Acknowledgments}

The authors thank the biostatisticians of the Department of Research Affairs for their statistical comments and analysis, as well as Dong-Su Jang, MFA, medical illustrator, Medical Research Support Section, Yonsei University College of Medicine, for his help with the figures.

\section{Authors' contributions}

NYK made substantial contributions to the design and conduct of the study, analysis of the data, and writing of the manuscript. TDK conceived of the study and participated in its design and coordination. SJB participated in data acquisition and its design. SHN made substantial contributions to the conduct of the study. JHH performed the statistical analysis. HL participated in data acquisition. KYL participated as the corresponding author and supervised the overall study and construction of the manuscript. All authors contributed to the manuscript, and have read and approved of the final manuscript.

\section{Competing Interests}

The authors have declared that no competing interest exists.

\section{References}

1. Wu $\mathrm{Y}$, Liu F, Tang $\mathrm{H}$, Wang $\mathrm{O}$, Chen $\mathrm{L}, \mathrm{Wu} \mathrm{H}$, Zhang $\mathrm{X}$, Miao J, Zhu M, Hu C et al: The analgesic efficacy of subcostal transversus abdominis plane block compared with thoracic epidural analgesia and intravenous opioid analgesia after radical gastrectomy. Anesthesia and analgesia 2013, 117(2):507-513.

2. Zheng X, Feng X, Cai XJ: Effectiveness and safety of continuous wound infiltration for postoperative pain management after open gastrectomy. World journal of gastroenterology 2016, 22(5):1902-1910.

3. Singh AP, Singh D, Singh Y, Jain G: Postoperative analgesic efficacy of epidural tramadol as adjutant to ropivacaine in adult upper abdominal surgeries. Anesthesia, essays and researches 2015, 9(3):369-373.

4. Zhu Z, Wang $\mathrm{C}, \mathrm{Xu} \mathrm{C}$, Cai Q: Influence of patient-controlled epidural analgesia versus patient-controlled intravenous analgesia on postoperative pain control and recovery after gastrectomy for gastric cancer: a prospective randomized trial. Gastric cancer : official journal of the International Gastric Cancer Association and the Japanese Gastric Cancer Association 2013, 16(2):193-200.

5. Manion SC, Brennan TJ: Thoracic epidural analgesia and acute pain management. Anesthesiology 2011, 115(1):181-188.

6. Manikian B, Cantineau JP, Bertrand M, Kieffer E, Sartene $R$, Viars P. Improvement of diaphragmatic function by a thoracic extradural block after upper abdominal surgery. Anesthesiology 1988, 68(3):379-386.

7. Freise $\mathrm{H}$, Van Aken HK: Risks and benefits of thoracic epidural anaesthesia. $\mathrm{Br}$ J Anaesth 2011, 107(6):859-868.

8. Moen V, Dahlgren N, Irestedt L: Severe neurological complications after central neuraxial blockades in Sweden 1990-1999. Anesthesiology 2004, 101(4):950-959.

9. Burlacu CL: PCEA vs. PCA for post-thoracotomy pain: is this any longer the question? Journal of postgraduate medicine 2008, 54(2):80-81.

10. Lee JH, Park JH, Kil HK, Choi SH, Noh SH, Koo BN: Efficacy of intrathecal morphine combined with intravenous analgesia versus thoracic epidural analgesia after gastrectomy. Yonsei medical journal 2014, 55(4):1106-1114.

11. Rawal N: Epidural technique for postoperative pain: gold standard no more? Regional anesthesia and pain medicine 2012, 37(3):310-317.

12. Hankin CS, Schein J, Clark JA, Panchal S: Adverse events involving intravenous patient-controlled analgesia. American journal of health-system pharmacy : AJHP : official journal of the American Society of Health-System Pharmacists 2007, 64(14):1492-1499.

13. Choi JB, Shim YH, Lee YW, Lee JS, Choi JR, Chang CH: Incidence and risk factors of postoperative nausea and vomiting in patients with fentanyl-based intravenous patient-controlled analgesia and single antiemetic prophylaxis. Yonsei medical journal 2014, 55(5):1430-1435.

14. Gerlach AT, Dasta JF: Dexmedetomidine: an updated review. Ann Pharmacother 2007, 41(2):245-252.

15. Ebert TJ, Hall JE, Barney JA, Uhrich TD, Colinco MD: The effects of increasing plasma concentrations of dexmedetomidine in humans. Anesthesiology 2000, 93(2):382-394.

16. Paris A, Tonner PH: Dexmedetomidine in anaesthesia. Curr Opin Anaesthesiol 2005, 18(4):412-418. 
17. Tufanogullari $\mathrm{B}$, White PF, Peixoto MP, Kianpour D, Lacour T, Griffin J, Skrivanek G, Macaluso A, Shah M, Provost DA: Dexmedetomidine infusion during laparoscopic bariatric surgery: the effect on recovery outcome variables. Anesthesia and analgesia 2008, 106(6):1741-1748.

18. Aho M, Erkola O, Kallio A, Scheinin H, Korttila K: Dexmedetomidine infusion for maintenance of anesthesia in patients undergoing abdominal hysterectomy. Anesthesia and analgesia 1992, 75(6):940-946.

19. Gurbet A, Basagan-Mogol E, Turker G, Ugun F, Kaya FN, Ozcan B: Intraoperative infusion of dexmedetomidine reduces perioperative analgesic requirements. Can J Anaesth 2006, 53(7):646-652.

20. Ge DJ, Qi B, Tang G, Li JY: Intraoperative Dexmedetomidine Promotes Postoperative Analgesia in Patients After Abdominal Colectomy: A Consort-Prospective, Randomized, Controlled Clinical Trial. Medicine 2015, 94(37):e1514.

21. Lin TF, Yeh YC, Lin FS, Wang YP, Lin CJ, Sun WZ, Fan SZ: Effect of combining dexmedetomidine and morphine for intravenous patient-controlled analgesia. Br I Anaesth 2009, 102(1):117-122.

22. Ren $\mathrm{C}$, Chi $\mathrm{M}$, Zhang $\mathrm{Y}$, Zhang $\mathrm{Z}, \mathrm{Qi}$ F, Liu Z: Dexmedetomidine in Postoperative Analgesia in Patients Undergoing Hysterectomy: A CONSORT-Prospective, Randomized, Controlled Trial. Medicine 2015, 94(32):e1348.

23. Ren C, Zhang X, Liu Z, Li C, Zhang Z, Qi F: Effect of Intraoperative and Postoperative Infusion of Dexmedetomidine on the Quality of Postoperative Analgesia in Highly Nicotine-Dependent Patients After Thoracic Surgery: A CONSORT-Prospective, Randomized, Controlled Trial. Medicine 2015, 94(32):e1329.

24. Nie Y, Liu Y, Luo Q, Huang S: Effect of dexmedetomidine combined with sufentanil for post-caesarean section intravenous analgesia: a randomised, placebo-controlled study. European journal of anaesthesiology 2014, 31(4):197-203.

25. Williamson A, Hoggart B: Pain: a review of three commonly used pain rating scales. Journal of clinical nursing 2005, 14(7):798-804.

26. Perkins FM, Kehlet H: Chronic pain as an outcome of surgery. A review of predictive factors. Anesthesiology 2000, 93(4):1123-1133.

27. Wightman JA: A prospective survey of the incidence of postoperative pulmonary complications. The British journal of surgery 1968, 55(2):85-91.

28. Latimer RG, Dickman M, Day WC, Gunn ML, Schmidt CD: Ventilatory patterns and pulmonary complications after upper abdominal surgery determined by preoperative and postoperative computerized spirometry and blood gas analysis. American journal of surgery 1971, 122(5):622-632.

29. Ferguson SE, Malhotra T, Seshan VE, Levine DA, Sonoda Y, Chi DS, Barakat RR, Abu-Rustum NR: A prospective randomized trial comparing patient-controlled epidural analgesia to patient-controlled intravenous analgesia on postoperative pain control and recovery after major open gynecologic cancer surgery. Gynecologic oncology 2009, 114(1):111-116.

30. Block BM, Liu SS, Rowlingson AJ, Cowan AR, Cowan JA, Jr., Wu CL: Efficacy of postoperative epidural analgesia: a meta-analysis. Jama 2003, 290(18):2455-2463.

31. Gritsenko K, Khelemsky Y, Kaye AD, Vadivelu N, Urman RD: Multimodal therapy in perioperative analgesia. Best practice \& research Clinical anaesthesiology 2014, 28(1):59-79.

32. Joshi GP: Multimodal analgesia techniques and postoperative rehabilitation. Anesthesiology clinics of North America 2005, 23(1):185-202.

33. Chen JY, Wu GJ, Mok MS, Chou YH, Sun WZ, Chen PL, Chan WS, Yien HW, Wen YR: Effect of adding ketorolac to intravenous morphine patient-controlled analgesia on bowel function in colorectal surgery patients--a prospective, randomized, double-blind study. Acta anaesthesiologica Scandinavica 2005, 49(4):546-551.

34. Yeh YC, Lin TF, Lin FS, Wang YP, Lin CJ, Sun WZ: Combination of opioid agonist and agonist-antagonist: patient-controlled analgesia requirement and adverse events among different-ratio morphine and nalbuphine admixtures for postoperative pain. Br J Anaesth 2008, 101(4):542-548.

35. Michelet P, Guervilly C, Helaine A, Avaro JP, Blayac D, Gaillat F, Dantin T, Thomas P, Kerbaul F: Adding ketamine to morphine for patient-controlled analgesia after thoracic surgery: influence on morphine consumption, respiratory function, and nocturnal desaturation. Br I Anaesth 2007, 99(3):396-403.

36. Clemente A, Carli F: The physiological effects of thoracic epidural anesthesia and analgesia on the cardiovascular, respiratory and gastrointestinal systems. Minerva anestesiologica 2008, 74(10):549-563.

37. Popping DM, Elia N, Van Aken HK, Marret E, Schug SA, Kranke P, Wenk M, Tramer MR: Impact of epidural analgesia on mortality and morbidity after surgery: systematic review and meta-analysis of randomized controlled trials. Annals of surgery 2014, 259(6):1056-1067.

38. Song Y, Shim JK, Song JW, Kim EK, Kwak YL: Dexmedetomidine added to an opioid-based analgesic regimen for the prevention of postoperative nausea and vomiting in highly susceptible patients: A randomised controlled trial. European journal of anaesthesiology 2016, 33(2):75-83. 